\title{
Color opponent receptive fields self-organize in a biophysical model of visual cortex via spike-timing dependent plasticity
}

\author{
Akihiro Eguchi $^{1 *}$, Samuel A. Neymotin ${ }^{2,3}$ and Simon M. Stringer ${ }^{1}$ \\ 1 Oxford Centre for Theoretical Neuroscience and Artificial Intelligence, University of Oxford, Oxford, UK \\ ${ }^{2}$ Department of Physiology and Pharmacology, Downstate Medical Center, State University of New York, New York, NY, USA \\ ${ }^{3}$ Department of Neurobiology, Yale University School of Medicine, New Haven, CT, USA
}

\section{Edited by:}

A. Ravishankar Rao, IBM Research, USA

\section{Reviewed by:}

James A. Bednar, University of

Edinburgh, UK

Yoonsuck Choe, Texas A\&M

University, USA

*Correspondence:

Akihiro Eguchi, Department of Experimental Psychology, Oxford Centre for Theoretical Neuroscience and Artificial Intelligence, University of Oxford, South Parks Road,

Oxford, Oxfordshire, OX1 3UD, UK e-mail: akihiro.eguchi@psy.ox.ac.uk
Although many computational models have been proposed to explain orientation maps in primary visual cortex (V1), it is not yet known how similar clusters of color-selective neurons in macaque V1/N2 are connected and develop. In this work, we address the problem of understanding the cortical processing of color information with a possible mechanism of the development of the patchy distribution of color selectivity via computational modeling. Each color input is decomposed into a red, green, and blue representation and transmitted to the visual cortex via a simulated optic nerve in a luminance channel and red-green and blue-yellow opponent color channels. Our model of the early visual system consists of multiple topographically-arranged layers of excitatory and inhibitory neurons, with sparse intra-layer connectivity and feed-forward connectivity between layers. Layers are arranged based on anatomy of early visual pathways, and include a retina, lateral geniculate nucleus, and layered neocortex. Each neuron in the V1 output layer makes synaptic connections to neighboring neurons and receives the three types of signals in the different channels from the corresponding photoreceptor position. Synaptic weights are randomized and learned using spike-timing-dependent plasticity (STDP). After training with natural images, the neurons display heightened sensitivity to specific colors. Information-theoretic analysis reveals mutual information between particular stimuli and responses, and that the information reaches a maximum with fewer neurons in the higher layers, indicating that estimations of the input colors can be done using the output of fewer cells in the later stages of cortical processing. In addition, cells with similar color receptive fields form clusters. Analysis of spiking activity reveals increased firing synchrony between neurons when particular color inputs are presented or removed (ON-cell/OFF-cell).

Keywords: brain modeling, visual cortex, neocortex, color, color selectivity, self-organizing color maps, self-organizing feature maps, STDP

\section{INTRODUCTION}

It has long been known that many neurons in primary visual cortex (V1) are tuned to exhibit preference to particular simple oriented line segments, forming orientation maps that capture the preferred orientation of neurons across the cortical surfaces (Hubel and Wiesel, 1962). Similarly, clusters of color-selective neurons in areas V1/V2 have been reported, as mapped with optical imaging and electrophysiological recordings (Landisman and Ts'O, 2002; Friedman et al., 2003; Xiao et al., 2003; Lu and Roe, 2008; Salzmann et al., 2012). While several computational studies have been conducted to explain the emergence of the orientation map (Somers et al., 1995; Choe and Miikkulainen, 1998; Paik and Ringach, 2011), only a few have been done over such patchy distribution of color selectivity within an area of V1/V2 (Bednar et al., 2005; Rao and Xiao, 2012). Barrow et al. (1996) have proposed a model for the formation of cortical blobs, regions in primary visual cortex that are densely stained by cytochrome oxidase (CO) (Livingstone and Hubel, 1984), using the Hebbian learning rule. This model reproduces receptive fields of neurons inside and outside CO blobs, and the results showed that neurons outside the blobs are selective for orientation while neurons inside the blobs are selective for color. However, the spatial organization of a large number of color-selective areas was not studied in their model. In this paper, we investigate the emergence of the spatial organization of color preference maps by developing a hierarchical neural network model that reflects anatomically faithful processing pathways and projections.

Physiological studies have shown that color information is first represented by the activity of specific types of photoreceptors and transmitted along specific fibers in the optic nerve (Komatsu, 1998). Visual signals leaving the eyes then reach the primary visual cortex via the lateral geniculate nucleus (LGN). LGN has multi-layered organization, and different color information is coded at specific layers (Chatterjee and Callaway, 2003). Although actual neural processing is not known, Komatsu and Goda (2009) theorized that a two-stage model can explain the transformation 
of color signal that takes place between photoreceptors and V1, resulting in forming the color selective neurons. At the first stage, signals from color opponent neurons are linearly summed with various combinations of weights, with the results rectified. This information is then propagated to neurons in the second stage where a further linear summation and rectification is performed.

Rao and Xiao (2012) have recently started investigating similar principles in computational simulations and successfully produced maps of orientation and color selectivity using anatomically realistic projections incorporating two color opponent channels and a luminance channel. However, this model used rate-coded neurons, which do not convey the precise times of action potentials or spikes emitted by cells. Various physiological studies have indicated that spiking dynamics can be important for the simulation and information processing (Sugase et al., 1999; Freiwald and Tsao, 2010). Although our current model does not investigate orientation selectivity, one of the aims of our study is to expand the focus in previous research (Bednar et al., 2005; Rao and Xiao, 2012) to see if it is possible to observe the spatial organization of color preference maps and spike-timing related phenomena such as ON/OFF selectivity using more physiologically realistic Hodgkin-Huxley $(\mathrm{HH})$ neuron models via Spike-Timing Dependent Plasticity (STDP).

Many neural networks are implemented with rate-coded neuron since it is observed that the mean firing rates of sensory neurons are correlated with the intensity of the encoded stimulus feature. For example, it is widely viewed that the information sent to the visual cortex by the retinal ganglion cells are encoded by the mean firing rates of spike trains generated with a Poisson process. A theoretical study conducted by Rullen and Thorpe (2001) showed that rate codes are optimal for fast information transmission but cannot account for the efficiency of information transmission between the retina and the brain; however, temporal structure of the spike train can be efficiently used to maximize the information transfer rate. This could therefore be an important feature that contributes to the development of neurons tuned to specific features.

Another benefit of our approach is that the precise firing times of spiking $\mathrm{HH}$ neurons allow investigating the temporal dynamics of information processing. Such investigations could include determining the role of temporal processing of $\mathrm{C} 1, \mathrm{C} 2$, and $\mathrm{L}$ channels in LGN (Chatterjee and Callaway, 2003), and selective representation of different stimuli by neuronal population synchronization (Evans and Stringer, 2013). In addition, spiking neurons allow incorporation of biologically plausible learning rules, such as STDP. A number of experiments (Markram et al., 1997; Bi and Poo, 1998) have reported that synaptic strength changes depending on presynaptic and postsynaptic spike time, and this mechanism has been extensively studied from a theoretical point of view (Gerstner et al., 1996; Abbott and Nelson, 2000).

Meanwhile, similar to the orientation maps and color maps, physiological studies have shown that various brain areas manifest a small-world structure, characterized by the presence of highly clustered neurons (Yu et al., 2008), and the factors leading to this organization have been investigated in several theoretical works (Shin and Kim, 2006; Kato et al., 2007, 2009; Basalyga et al., 2011). In the present study, we were particularly interested in whether such small-world structures could evolve from a network whose weights were initialized randomly, after learning with natural images.

We speculated there would be difficulty in the development of such cells since the representation of color is more complex than oriented bars. However, with this model, we hypothesized that the response patterns of neurons in the output layer (layer 5 of V1) would develop heightened responses to specific colors solely due to learning taking place during exposure to multiple image patches extracted from natural images of indoor scenes used in Quattoni and Torralba (2009), as a result of integrating different color opponent signals that occurred at different levels of the network. We also hypothesized that the learning would allow for a distribution of neurons that were tuned to similar color input with spatial clustering, where neurons within the cluster had heightened synaptic weights, relative to neurons outside of the cluster.

\section{MATERIALS AND METHODS MODEL \\ Architecture}

The model is composed of nine layers of neurons which are organized into five hierarchical areas: photoreceptor layers (R, G, B), lateral geniculate nucleus (LGN) layers (L, C1, C2), V1 layer 4 (L4), V1 layer 2/3 (L2/3), and V1 layer 5 (L5). The dimensions of each layer are shown in Table 1, and the total number of neurons is thus 5700 .

Each color input presented to the network is first decomposed into an RGB representation (range: $0-1$ ) in digital images to be consistent with the trichromatic color vision in primates as a result of S, M, and L cones (Rowe, 2002) (Figure 1). The degree of each input is represented as different spiking frequencies of photoreceptors with $10 \%$ of random noise. To be consistent with physiology, a stimulus that a human would perceive as red activates the green channel very strongly as well. The frequency of each cone is determined as follow:

- $S_{\mathrm{freq}}=40[\mathrm{~Hz}] \times B$

- $M_{\text {freq }}=40[\mathrm{~Hz}] \times(G+R \times 0.7+B \times 0.25) /(1+0.7+$ $0.25)$

- $L_{\mathrm{freq}}=40[\mathrm{~Hz}] \times(R+G \times 0.7+B \times 0.25) /(1+0.7+0.25)$

Specific combinations of the decomposed color signals are then projected to cells in LGN. The projections reflect the physiological findings that reported different characteristics in different layers of LGN (Shapley et al., 1981). Specifically, as later studies

Table 1 | Dimensions of each layer.

\begin{tabular}{lc}
\hline Layer & Dimensions (number of cells) \\
\hline V1 layers (L4, L2/3, L5) & $30 \times 30$ \\
LGN layers (C1, C2, L) & $30 \times 30$ \\
Photoreceptor layers (L, M, S) & $10 \times 10$
\end{tabular}


revealed, different layers of LGN receive different visual information via optic nerves and show different functionality, forming a luminance channel (L) and two opponent color channels, comprising red-green $(\mathrm{C} 1)$ and blue-yellow (C2) channels as follows (Casagrande, 1994; Goda et al., 2009; Rao and Xiao, 2012):

- Magnocellular (MC) pathways: luminance channel $L=R+G$

- Parvocellular (PC) pathway: red/green opponent channel $C 1=R-G$

- Koniocellular (KC) pathway: blue/yellow opponent channel $C 2=(R+G)-B$

Physiological studies also report that while the MC and PC pathways project their output to $\mathrm{V} 1 \mathrm{~L} 4$, the $\mathrm{KC}$ pathway terminates in V1 L2/3 (Chatterjee and Callaway, 2003), and many neurons in $\mathrm{L} 2 / 3$ project excitatory connections to the neurons in V1 L5 (Douglas and Martin, 2007). Our model incorporates this anatomical architecture (Figure 1). Physiological evidence indicates that there is heavy feedback from V1 to LGN (from layer 6) and the thalamic reticular nucleus is involved in both the feed-forward and feedback pathways, and data also suggests that retinal ganglion cells have widely different spatial extent; however, these are beyond the scope of this paper and are not explicitly modeled.

\section{Synaptic connections}

Convergent connections are established to each neuron from a topologically corresponding region of the preceding layer, leading to an increase in the receptive field size of neurons through the visual processing areas, which reflects the known physiology of the primate ventral visual pathway (Pettet and Gilbert, 1992; Freeman and Simoncelli, 2011). While synaptic weights between the photoreceptor layers and LGN layers are kept static, the

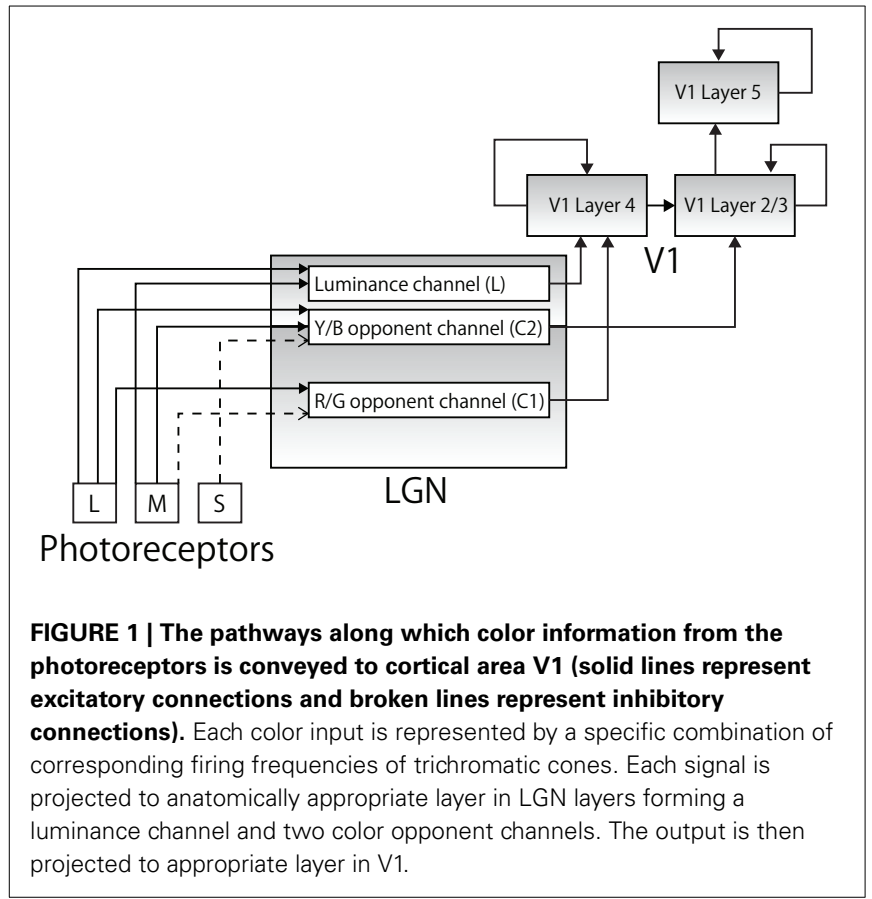

weights of other feed-forward connections are learned through visually guided learning.

Each feed-forward connection requires a $1 \mathrm{~ms}$ delay for signal transmission. Each neuron also establishes lateral short-range excitatory connections and long-range inhibitory connections, forming a Mexican-hat spatial profile (Figure 2). Whether this kind of lateral connectivity exists at the anatomical level is debatable (Martin, 2002; Kang et al., 2003; Hopf et al., 2006; Adesnik and Scanziani, 2010), since a detailed microcircuitry map at the neuron-to-neuron level is not currently available. However, we incorporated this architecture to (1) be consistent with a previous model by Rao and Xiao (2012) and (2) to abstract the function exhibited by this kind of architecture (Kang et al., 2003; Neymotin et al., 2011b). Further experimental work that details the wiring of cortical microcircuitry may reveal whether these considerations were justified (Alivisatos et al., 2013). The synaptic delay is $1 \mathrm{~ms}$ for the excitatory connections and $4 \mathrm{~ms}$ for the inhibitory connections.

\section{Learning mechanism (STDP)}

While synaptic weights at the connections between photoreceptor layers and LGN layers were fixed, weights in all the other feedforward connections were plastic. Each synaptic weight in the model was learned using STDP, where Long-term potentiation (LTP) is caused if the pre-synaptic spike precedes the postsynaptic spike, and Long-term depression (LTD) is caused if the spike timing is in the opposite order. The degree of the modification depends on how close the two spikes are in time (Bi and Poo, 1998) as follows:

$$
\Delta w= \begin{cases}\operatorname{LR} \times \exp \left(\frac{-\left(t_{\text {post }}-t_{\text {pre }}\right)}{p_{\text {tau }}}\right) & \text { if }\left(t_{\text {post }}-t_{\text {pre }}\right)>0 \\ -\operatorname{LR} \times \exp \left(\frac{t_{\text {post }}-t_{\text {pre }}}{d_{\text {tau }}}\right) & \text { if }\left(t_{\text {post }}-t_{\text {pre }}\right)<0\end{cases}
$$

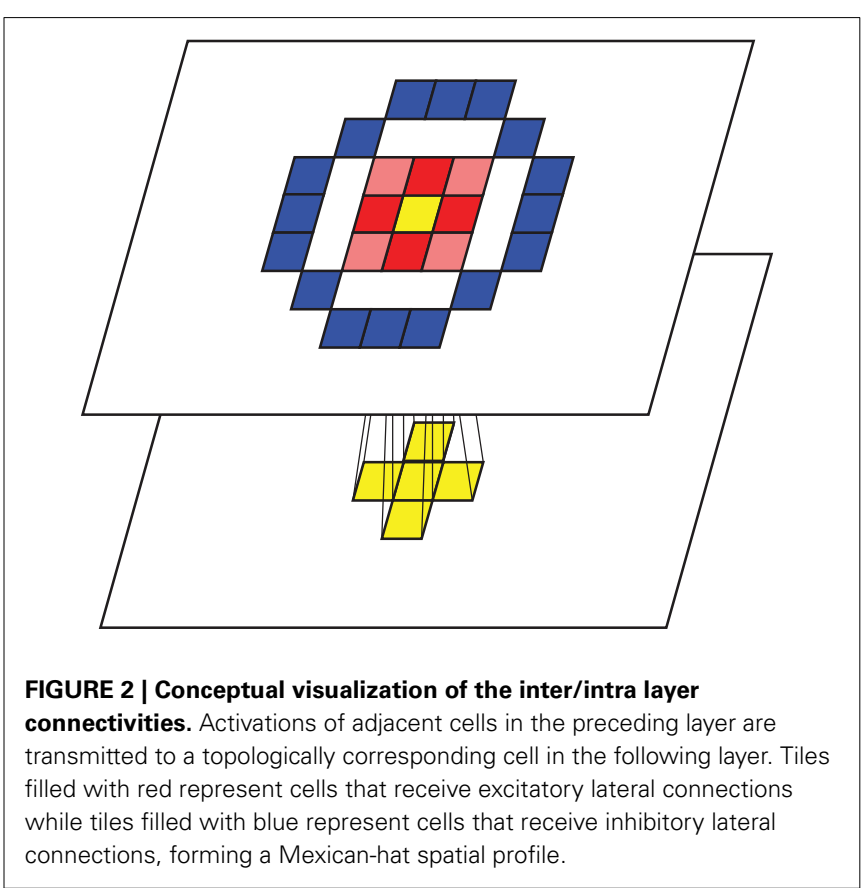


where LR is a learning rate, $t_{\text {pre }}$ is the time when presynaptic cell becomes activated, $t_{\text {post }}$ is the time when postsynaptic cell becomes activated, and $p_{\mathrm{tau}} / d_{\mathrm{tau}}$ controls the range of the influence. The curve generated by this function is show in Figure 3. Weights are originally randomly assigned within a fixed range, and after every iteration, weights in the same layers are normalized so that the mean of all the values are always kept in the middle of the pre-specified range, and also to prevent runaway excitation (Neymotin et al., 2011a, 2013; Rowan and Neymotin, 2013). Neurophysiological evidence for synaptic weight normalization is provided by Royer and Paré (2003).

\section{Neuron model}

Model neurons utilized the standard parallel conductance model with Hodgkin-Huxley dynamics for generating action potentials. Neurons consisted of a single compartment (diameter of $30 \mu \mathrm{m}$, length of $10 \mu \mathrm{m}$, axial resistivity of $100 \Omega \mathrm{cm}$ ). The rate of change of a neuron's voltage $(\mathrm{V})$ was represented as $-C_{m} \frac{d V}{d t}=g_{\text {pas }}\left(v-e_{\text {leak }}\right)+i_{\text {syn }}+i_{N a}+i_{K}$, where $C_{m}$ is the capacitive density $\left(10 \mu \mathrm{F} / \mathrm{cm}^{2}\right), i_{\text {syn }}$ is the summed synaptic current, and $i_{\mathrm{Na}}$ and $i_{K}$ represent the $\mathrm{Na}^{+}$and $\mathrm{K}^{+}$currents from the Hodgkin-Huxley channels. $g_{\text {pas }}$ represents the leak conductance $(0.001 \mathrm{nS})$, which was associated with a reversal potential, $e_{\text {leak}}$, of $0 \mathrm{mV}$.

Synapses were modeled using an instantaneous rise of conductance, followed by exponential decay with specified time-constant, $\tau$. For excitatory synapses, we utilized AMPA synapses $\left(\tau=5 \mathrm{~ms}, e_{\text {rev }}=0 \mathrm{mV}\right)$, while for inhibitory GABA synapses $\left(\tau=10\right.$ and $\left.e_{\text {rev }}=-80\right)$. Synaptic currents followed $i_{\text {syn }}=g\left(v-e_{\text {rev }}\right)$, where $v$ is the membrane potential, and $e_{\text {rev }}$ is the reversal potential associated with the synapse.

\section{Software}

Simulations were run using the NEURON simulation environment with the Python interpreter, multithreaded over 16-32 threads (Hines and Carnevale, 2001; Carnevale and Hines, 2009; Hines et al., 2009). Simulation is posted on ModelDB (https://senselab.med.yale.edu/ModelDB/ShowModel.
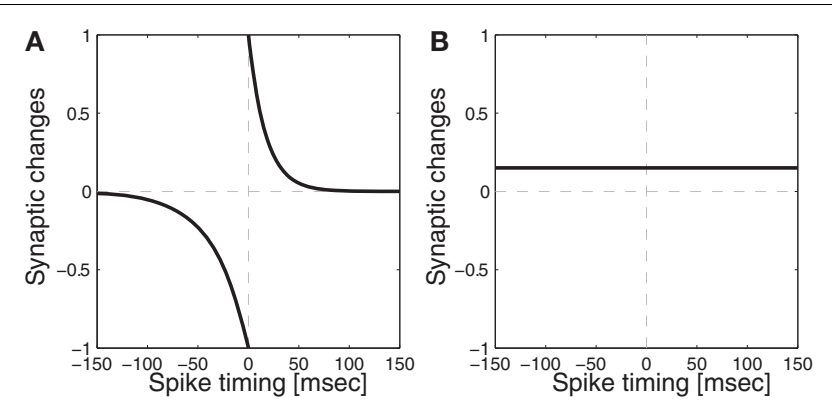

FIGURE 3 | Synaptic modification functions with/without Spike-Timing Dependent Plasticity (STDP). (A) Function with STDP: temporal windows for depression $\left(d_{\text {tau }}=34 \mathrm{~ms}\right.$ ) and potentiation ( $p_{\text {tau }}=17 \mathrm{~ms}$ ) used for spike-timing dependent plasticity where the equation is given in Equation (1) (B) Function without STDP: the synaptic weights are potentiated whenever both pre and post synaptic neurons become activated during the training time for $300 \mathrm{~ms}$. asp?model=152197) (Hines et al., 2004). Simulations were run on Linux on a $2.93 \mathrm{GHz}$ 16-core Intel Xeon CPU X5670. A $300 \mathrm{~ms}$ simulation ran in approximately $30 \mathrm{~s}$.

\section{DATA ANALYSIS METHODS Clustering}

In order to quantify the degree of clustering of the activations in the network, a clustering coefficient $C$ is calculated based on the responses among different color inputs at every training iteration as follows (modified from Kato et al., 2007):

$$
\begin{aligned}
C & =\frac{1}{n \text { Cells } \times n \text { Stims }} \sum_{s=1}^{n \text { Stims }} \sum_{i=1}^{n \text { Cells }} C_{s, i} \\
C_{s, i} & =\frac{\sum_{l=1}^{k_{s, i}} \sum_{m=l+1}^{k_{s, i}}\left(\mathrm{FR}_{s, l} \times \mathrm{FR}_{s, m}\right)}{{ }_{k i} \mathrm{C}_{2}}
\end{aligned}
$$

where $n$ Cells is the number of neurons in a network; $n$ Stims is the number of stimuli during the testing; $\mathrm{FR}_{s, i}$ is the firing rates of the cell $i$ when exposed to a stimulus $s$; $k_{i}$ sets the nearby neurons from the $i$-th neuron for the analysis. We use $9(3 \times 3)$ for the $k$ value.

\section{Single-cell information}

A single cell information measure was applied to individual cells to measure how much information is available from the responses of a single cell about which color input is present. The amount of color specific information that a certain cell transmits is calculated from the following formula:

$$
I(s, \vec{R})=\sum_{r \in \vec{R}} P(r \mid s) \log _{2} \frac{P(r \mid s)}{P(r)}
$$

Here $s$ is a particular color and $\vec{R}$ is the set of responses of a cell to the set of color stimuli, which are composed of eight colors slightly varied the RGB values of original color by $\pm 1 \%$. This is based on the assumption that the same set of tuned cells will still respond to slightly variant colors and is to well differentiate the tuned cells from randomly responding cells. The maximum information that an ideally developed cell could carry is given by the formula:

$$
\text { Maximum cell information }=\log _{2}(n \times p) \text { bits }
$$

As eight different sets of colors (combination of 0 and 1 for each RGB value) are used in this analysis, the maximum information could be carried in this analysis is 3 .

\section{Multiple-cell information}

A multiple-cell information measure was used to quantify the network's ability to tell which stimulus is currently exposed to the network based on the set of responses, $R$, of a sub-population of cells, $\vec{C}$, as following formula with details given by Rolls and Milward (2000). 


$$
\begin{aligned}
I_{\vec{C}}\left(S, S^{\prime}\right) & =\sum_{s, s^{\prime}} P\left(s, s^{\prime}\right) \log _{2} \frac{P\left(s, s^{\prime}\right)}{P(s) P\left(s^{\prime}\right)} \\
P\left(s^{\prime}\right) & =\sum_{s \in S} P\left(s^{\prime} \mid R_{\vec{C}}(s)\right) \times P\left(R_{\vec{C}}(s)\right) \\
P\left(s, s^{\prime}\right) & =P\left(s^{\prime} \mid R_{\vec{C}}(s)\right) \times P\left(R_{\vec{C}}(s)\right)
\end{aligned}
$$

Here, $S$ represents the set of the stimuli presented to the networks, and $\vec{C}$ defines the set of cells used in the analysis, which had as single cells the most information about which color input was present. From the set of cells $\vec{C}$, the firing responses $R_{\vec{C}}(R=r(c) \mid c \in \vec{C})$ to each color in $S$ are used as the basis for the Bayesian decoding procedure as follows:

$$
\begin{aligned}
P\left(s^{\prime} \mid R_{\vec{C}}\right) & =\frac{P\left(s^{\prime}\right) \prod_{c \in \vec{C}} P\left(R_{c}\left(s^{\prime}\right) \mid s^{\prime}\right)}{\sum_{s^{\prime \prime} \in S} P\left(s^{\prime \prime}\right) \prod_{c \in \vec{C}} P\left(R_{c}\left(s^{\prime \prime}\right) \mid s^{\prime \prime}\right)} \\
\left.P\left(R_{c}(s)\right) \mid s^{\prime}\right) & =\frac{\sum_{t=1}^{n \operatorname{Trans}} p d f\left(R_{c}(s, t), \bar{R}_{c}\left(s^{\prime}\right), S D_{c}\left(s^{\prime}\right)\right)}{n \operatorname{Trans}}
\end{aligned}
$$

where $n$ Trans defines the number of possible transforms; in this case, similar but slightly different colors, and pdf computes the probability density function at firing response of a subset of cells when exposed to a stimulus $s$ at $t$ th transforms using the normal distribution with their mean and standard deviation.

\section{RESULTS}

The results described in this study used a network model trained with various small color image patches extracted from original natural images of indoor scenes used in Quattoni and Torralba (2009). The size of the photoreceptor layer in our model is $10 \times 10$ pixels while the size of original images was an average of $504.1 \times 658.4$ pixels (112 images). The training sessionconsisted of 2000 iterations, where 2000 different $10 \times 10$ image patches were extracted from the set of images. This was designed as an abstraction of natural viewing, where eyes saccade, and the activation of photoreceptors corresponds to visual inputs bounded by their range of view.

\section{LEARNING PRODUCES SPATIAL CLUSTERING}

During the training, synaptic efficacy between each of two layers progressed from a uniform distribution at the initial state toward a binary distribution where only a limited number of synaptic connections were highly strengthened or weakened (Figure 4). This convergence toward an bimodal equilibrium state is consistent with other self-organizing modeling work with STDP (Song et al., 2000; Kato et al., 2009; Basalyga et al., 2011). Contrary, physiological studies have shown that synaptic weights tend to have unimodal distributions with a positive skew (Barbour et al., 2007). Barbour et al. (2007) raised a possible reconciliation with the bimodal distributions of modeling with such experimental data, given that the dendritic distribution of synaptic weights may have a wide range of values, due to electrotonic filtering effects. However, in order to explore this possibility, further investigation will be required.

Investigation into the firing count of each neuron to different color inputs shows that the weight convergence resulted in development of clustered responses in the networks (Figure 5). A comparison between the results with the weight distribution plots in Figure 4 shows that even though the average weight was kept constant, neuronal firing activity became more prominent and deviated after the training; it was sparse (average rate of $2.165 \mathrm{~Hz}$ with standard deviation of 0.874 ) prior to learning, but after 2000 iterations of $300 \mathrm{~ms}$ exposure to image patches extracted from natural indoor images, the network developed different clustered firing patterns of neurons (average rate of $3.966 \mathrm{~Hz}$ with standard deviation of 1.169 ) for eight different

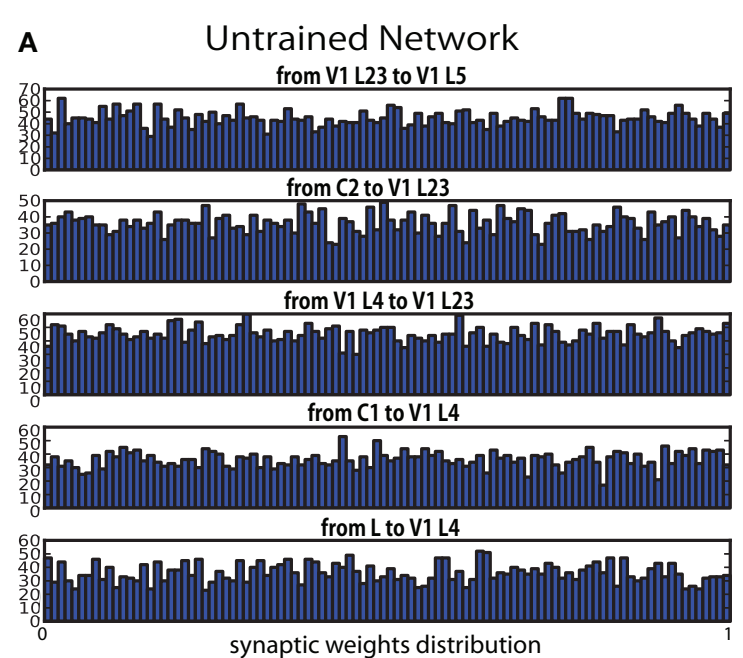

FIGURE 4 | Synaptic weight distribution at synapses before (0 iteration) and after (2000 iterations) the learning. Weights are initialized randomly. After every iteration, connection weights between the two layers are normalized so that the mean of all the values are

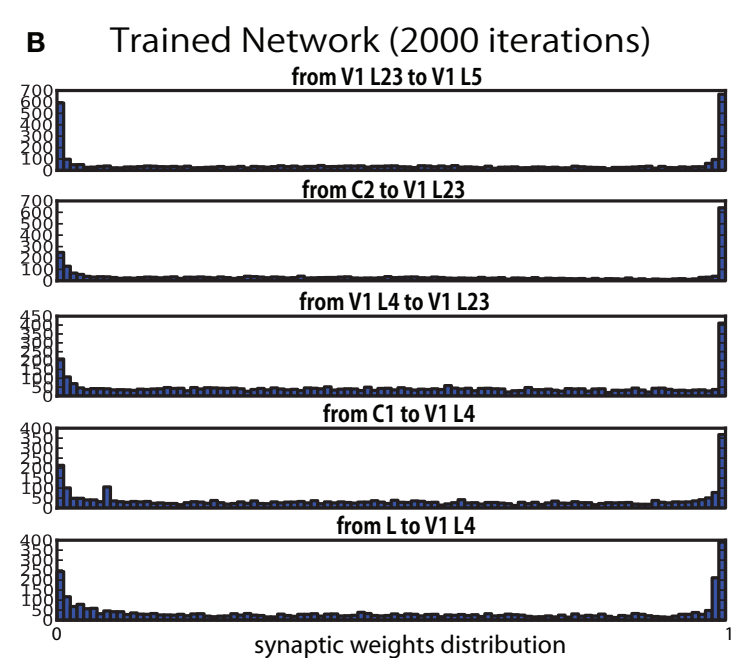

kept in the middle of the pre-specified range, and to prevent runaway excitation. The graphs show that the weights converged over the course of the training sessions. (A) Untrained network. (B) Trained network. 


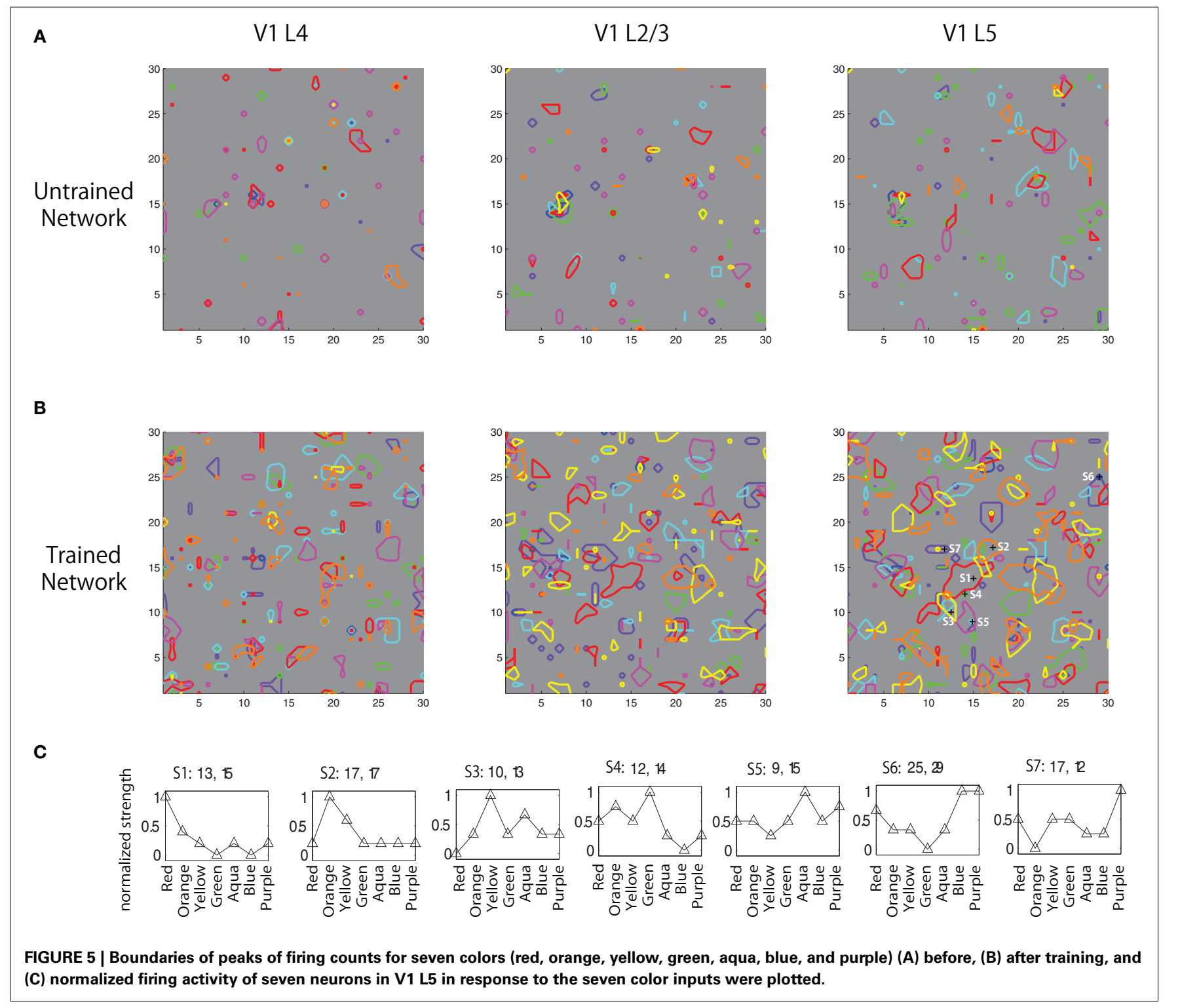

color inputs (red, orange, yellow, green, aqua, blue, purple, and pink).

In Figures 5A,B, the boundaries of peaks of firing counts for seven different colors (red, orange, yellow, green, aqua, blue, and purple) before and after training are plotted. The result shows that the training resulted in developing color selective clustered responses. Normalized firing activity of seven neurons in V1 L5 were recorded and plotted in Figure 5C. These results failed to show a clear spatial shift of the activation with gradual change of color inputs as reported in Xiao et al. (2003); however, the results revealed gradual changes of firing patterns according to changes of input colors, which is partially consistent with the physiological findings. This also shows that some cells show higher selectivity than others at responding to similar colors. This is likely due to the fact that the color representation takes a specific combination of three continuous values of RGB.
Depending on the trained weights, activations of some neurons may only be influenced by one or two of the three values, and the activation patterns also vary due to different combinations of those values and influences from other nearby neurons.

We calculated a clustering coefficient $[C$; Equations $(3,3)]$ to assess the effectiveness of training in producing spatial clustering within the network. Figure 6 shows $C$ of V1 L4, V1 L2/3, V1 L5, as well as of V1 L5 trained with Hebb-like learning rule, plotted as a function of training iteration. The result demonstrates that the networks trained with STDP rule gradually increases clustering coefficients as training proceeds while the network trained with Hebb-like learning rule remains relatively low clustering coefficient.

The emergence of clustering may be explained by the lateral excitatory connections described in section 2.1.2. When a specific neuron becomes activated, the signal is propagated to 


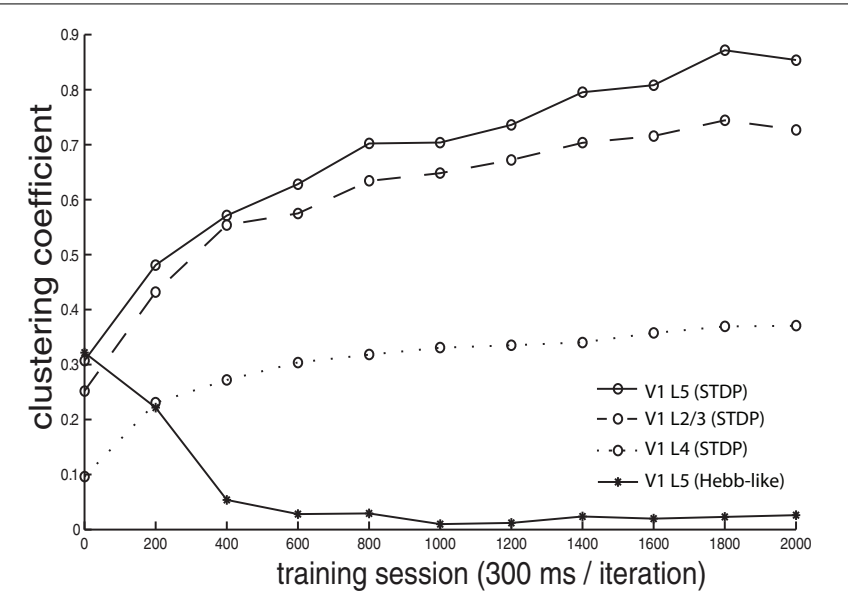

FIGURE 6 | Clustering coefficient dynamics during the training. The clustering coefficients $C$ of $V 1 L 4, L 2 / 3$, and $L 5$, where the networks were trained with STDP, were calculated by the equation given in Equations $(3,3)$ and plotted by dotted line, dashed line, and solid line with circle markers, respectively. Additionally, the clustering coefficients of V1 L5 when the network was implemented with Hebb-like rule is plotted by the line with asterisk markers. The result demonstrates that the networks trained with STDP rule gradually increases clustering coefficients as training proceeds while the network trained with Hebb-like learning rule remains relatively low clustering coefficient.

the neighboring neurons making them more likely to become activated as well. Once the neighboring cell reaches a threshold and becomes activated, synaptic connections convergent onto the cell from recently activated cells in the preceding layer become strengthened via STDP. Repetitions of this process are likely to be the cause of the development of the clustered responses of cells. This phenomenon should be prominent only among nearby cells because of lateral propagation delays and long-range lateral inhibition.

The precise temporal dependence of STDP is crucial for the clustering learning process. Activation of neurons are laterally propagated within layers but with a specified delay. Therefore, temporal differences of the activations between the source in the preceding layer and the targets in the following layers become large as the signal is propagated. As a result, the degree of LTP decays as the differences become large, and LTD is turned on if the post-synaptic activation timing becomes closer to the next presynaptic activation, thus forming the distinct clustering responses in the networks.

In order to confirm the importance of spike-timing in forming color receptive field clustering, we ran a control simulation, using a Hebbian plasticity synaptic learning rule, which does not take into account the timing of pre- and post-synaptic neuronal spiking (Figure 3B). After learning with this Hebbian plasticity rule, the clustering coefficient value remained low (Figure 6 lines with asterisks) relative to the results in the network trained with STDP. This underlines the importance of STDP in developing clustering in our model.

In addition, our model shows that the clustering coefficient in higher layers tended to be larger. This observation makes us expect information to gradually change in the different layers, and this assumption has been confirmed in the next section.

\section{SELECTIVITY OF THE RESPONSES}

In order to identify how the learned connectivity shaped output neuron sensitivity to stimuli, the techniques of Shannon's information theory were employed (Rolls and Treves, 1998). If the responses $r$ of a neuron carry a high level of information about the presence of a particular color stimulus $s$, this implies that the neuron will respond selectively to the presence of that color. Two information measures were used to assess the ability of the network to develop neurons that are selective to the presence of a particular color by measuring single cell and population information (see sections 2.2.2, 2.2.3). Since eight different sets of colors (red, orange, yellow, green, aqua, blue, purple, and pink) are used in this analysis, the maximum information carried in this analysis is 3 bits.

Figure 7A shows the single cell information analysis as plotted in rank order according to maximum information each cell carries for a specific stimulus. The results compare the information distribution of each layer in the trained network and of the final layer (V1 L5) in the untrained and trained network. The results demonstrate that neurons in the trained network generally carry more single-cell information.

While useful in assessing the tuning properties of a particular neuron, the single-cell information measure cannot provide mutuality of the responses; if all cells learned to respond to the same color input (according to the single-cell measure) then there would be relatively little information available about the whole set of color stimuli $S$. To address this issue, we used a multiple-cell information measure, which assesses the amount of information that is available about the whole set of color inputs from a population of neurons (see section 2.2.3).

In Figure 7B, the multiple cell information measures are plotted according to the number of cells used in the analysis. The result shows that the trained network conveys more color specific information than the untrained network. More interestingly, we found that the amount of color specific mutual information reaches a maximum with fewer neurons in the higher layers: 13 neurons in L4, 10 neurons in L2/3, and 8 neurons in L5. This analysis indicates that estimations of the input colors can be done using the output of fewer cells in the later stages of cortical processing.

More precisely, the total amount of mutual information (across a layer) can not increase through further processing as the Data Processing Inequality (DPI) states - it can only be preserved or lost. In other words, if all the information from all cells in each of the two layers was added up, it will decrease in the higher layer. However, our specific information measure explained in section 2.2.3 can increase for particular cells, as they become more selective throughout the layers. In this case, some of that information has shifted into different cells, and so all stimuli can now be represented with fewer neurons, allowing for fewer required cells to convey maximum information. Our information measure therefore improves, showing that the cells are becoming more tuned, even though the total information in the layer has decreased. 


\section{ON-AND OFF-CELLS}

The firing pattern of each cell in response to turning a stimulus $\mathrm{ON}$ and OFF was also investigated. During this testing procedure, eight different colors (red, orange, yellow, green, aqua, blue, purple, and pink) are presented for $240 \mathrm{~ms}$, followed by $60 \mathrm{~ms}$ of no visual input presentation, and the voltage level of each neuron is recorded. In order to find if any neuron developed ON/OFF sensitivities during training with similar properties to those found in V1/V2 in vivo (Michael, 1978; Friedman et al., 2003), from each recorded voltage dynamics, the 30 neurons which responded the most during the first $60 \mathrm{~ms}$ and the last $60 \mathrm{~ms}$ were selected to be plotted in Figure 8. Similar to the physiological findings, we found both ON- and OFF-cells for each different color input, where populations of neurons showed a burst of firing just after a presentation or removal of a color input.

Also, further analysis revealed that some of those cells displayed the temporal color opponent property as reported in Friedman et al. (2003). Figure 9A shows two types of such cells: Red-ON/Green-OFF cells and Yellow-ON/Blue-OFF cells.

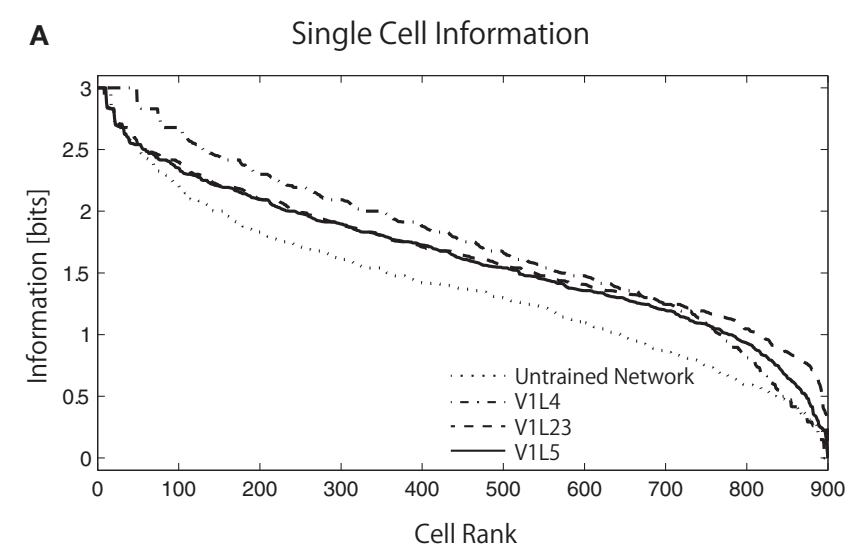

FIGURE 7 | Single-cell/Multiple-cell information analysis of V1 L5. Solid lines represents trained networks while dotted line represents naive network. (A) The single cell information measure are plotted in rank order according to how much information they carry. The result show that the maximum information each cell carry drops rapidly in the naive network while most of the cells in the trained network carry relatively higher amount of information. (B) The multiple cell information measures are plotted according to the
B

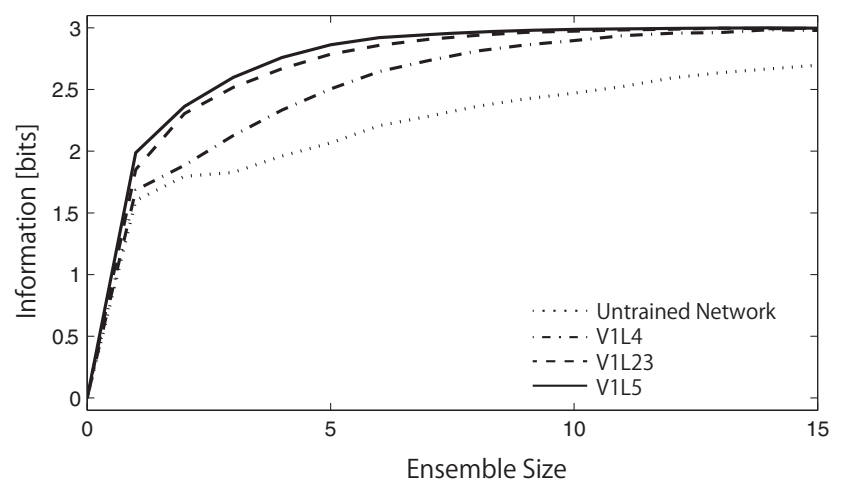

number of cells used in the analysis to visualize the mutuality of the responses. The information that the trained network carries reaches maximum mutual discriminability with 3 bits of information with around 8 neurons while the information that the naive network carries does not reach this point with 15 neurons. This result also shows that fewer neurons are required to represent all stimuli in the higher layers, as the information measure improves from $\mathrm{L} 4 \rightarrow \mathrm{L} 2 / 3 \rightarrow \mathrm{L} 5$.
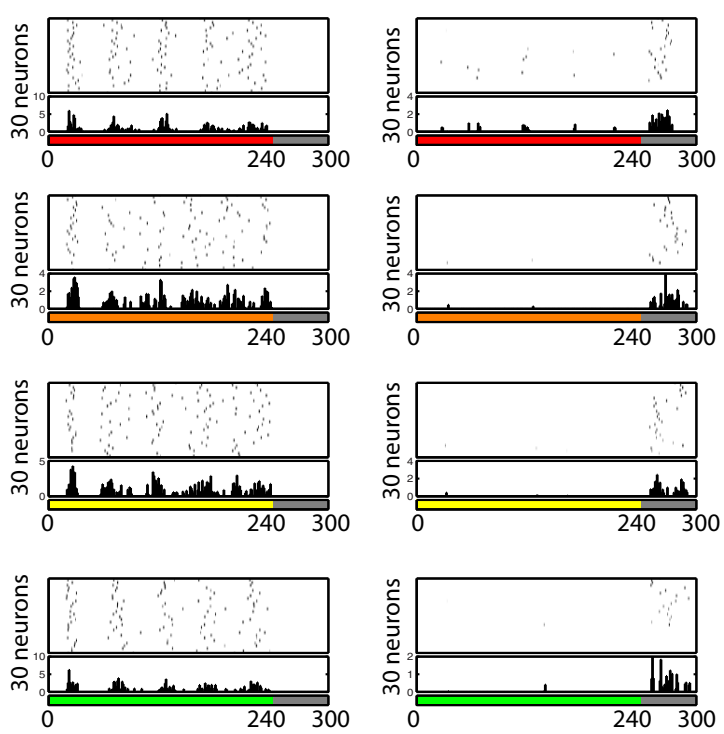

FIGURE 8 | Firing activity of 30 neurons in V1 L5, which responded vigorously when color input is presented or removed, from each experiment. The color bars under each raster plot represent times at which
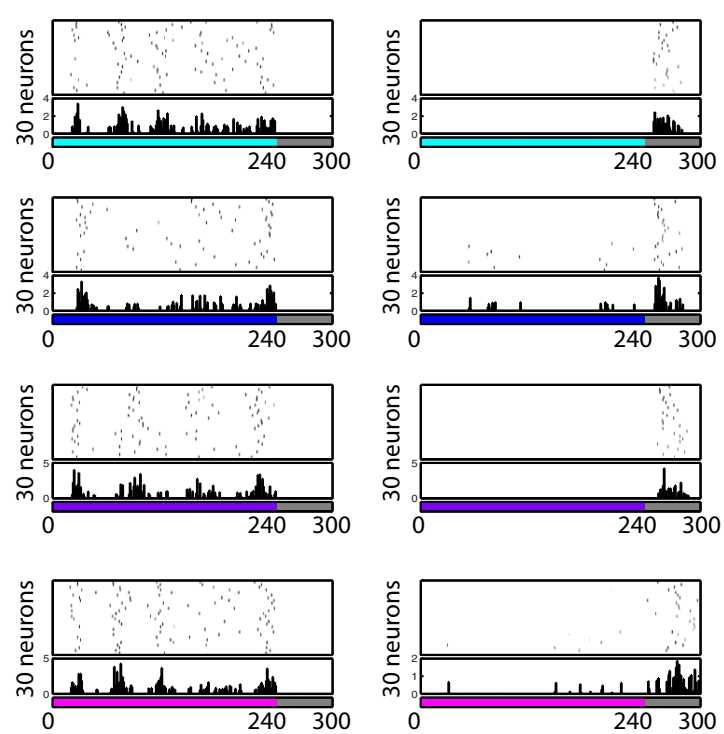

colors are presented to the neurons (each color is presented for $240 \mathrm{~ms}$ and removed). From these results, we found that many neurons exhibit the characteristics of ON/OFF-cells in the trained network. 


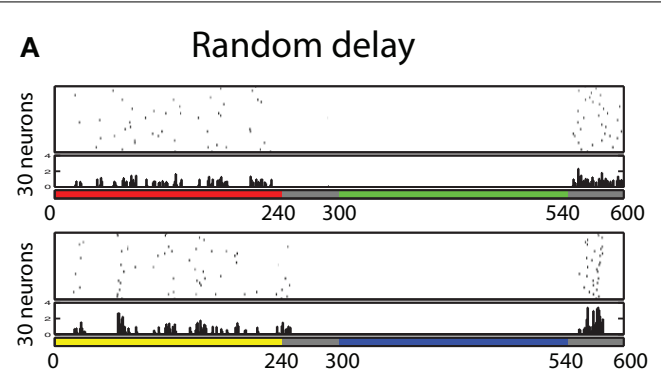

FIGURE 9 | Firing responses of neurons in the V1 L5, which shows temporal color opponent ON/OFF responses. Figure on the top shows 30 neurons that responds highly when color red is presented and when color green is removed. Figure on the bottom shows 30 neurons that responds
In order to find such cells, we first identify 100 cells that show Red-ON (or Yellow-ON) property, and then chose 30 cells from the subset that show Green-OFF (or Blue-OFF) property.

Neurons in the layers are exposed to different colors in natural images during the training, so the development of ON-cells which exhibit specific responses to specific inputs can be explained with standard feed-forward competitive learning principles (Rolls and Treves, 1998). In contrast, the development of OFF-cells are due to the lateral inhibitory connections emitted by ON-cells: suppose there are $\mathrm{ON}$-cells that were tuned to the color red. If red is presented to the network, these ON cells become activated making surrounding cells that receive inhibitory synaptic connections from the ON cells less likely to become activated. When the color input is removed, ON-cells stop activating. As a result, the surrounding cells are no longer suppressed by the ON-cells, demonstrating their being OFF-cells.

However, the question is where the OFF-cells receive excitatory input to enable them to remain activated after the removal of the color input. In other words, there should be some mechanism where ON-cells immediately stop receiving excitatory input while OFF-cells keep receiving excitatory input, even after the removal of the color input. This may be caused by the differences in firing timing of different input cells as explained in Figure 10.

In our model, the maximum activation frequency of input cells was set to $40 \mathrm{~Hz}$ ( $25 \mathrm{~ms}$ interspike interval), which is gamma oscillations which are widespread in the visual cortex. Also, different input cells have different randomly determined delays from the input cell receiving color input to its firing, which is reflected in their firing timings. As shown in Figure 10, suppose the spike timing of an input cell $\mathrm{A}$ is $24 \mathrm{~ms}$ earlier than another input cell B. This means that there is at most $24 \mathrm{~ms}$ difference between the final spike timing of cell A and the timing of cell B before the removal of the color input. This $24 \mathrm{~ms}$ difference will result in giving a chance for the OFF-cell that receives most of the inputs from the input cells such as B to become activated after an ON-cell that happens to receive most of the inputs from the input cells such as A stops activating inhibitory signals.

In order to confirm the importance of the delay for the development of such ON/OFF cells, we have also trained the same network without randomly determined delays from the input cell receiving color input to its firing timings. Figure $9 \mathbf{B}$ shows the

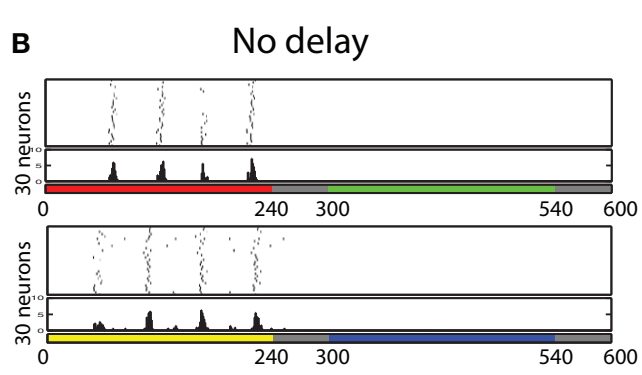

highly when color yellow is presented and when color blue is removed. This result shows that in the trained network, we found Yellow-ON/Blue-OFF cells and Red-ON/Green-OFF cells as reported in physiological experiments. (A) Random delay. (B) No delay

firing activities of each 30 neurons selected by the same procedure used to find Red-ON/Green-OFF cells and Yellow-ON/Blue-OFF cells earlier. The results show that in the network that employed inputs without randomized delays, we failed to find Green-OFF and Blue-OFF cells within each subset of 100 Red-ON cells and Yellow-ON cells. This result indicates that the randomized delay plays an important role for the development of the OFF cells.

In animal V1, much of the ON and OFF component of the responses are thought to be inherited from similar properties of LGN and RGC cells. Therefore, we are not expecting that onset and offset transients arise in V1 alone. However, our results suggested the possibility of multiple mechanisms that impact the firing times of these cells. 


\section{DISCUSSION}

In this study, we have developed a model of early visual processing of colors including the pathway beginning at photoreceptors and terminating in the fifth layer of V1. We have incorporated anatomically accurate projections of signals between layers and the biologically plausible learning of synaptic weights based on STDP using Hodgkin-Huxley models of neuronal dynamics.

We have successfully shown that the networks gradually develop clustered firing activity of neurons during training (section 3.1). Information analysis based on averaged firing rates of each neuron also confirmed development of neuronal color selectivity after the training (section 3.2). Our results also indicated that populations of neurons can provide reliable predictions of the input color presented to the retina. Interestingly, the color information measure by multiple-cell information analysis rises more rapidly with fewer cells from L4 $\rightarrow$ L2/3 $\rightarrow$ L5, suggesting that layered neocortical architecture may enable it to boost important information. We also found that if the synaptic weights in the network were learned via a Hebbian plasticity rule, the level of clustering coefficient remained low relative to the results in the network trained with STDP.

However, the question is why other models without STDP, including the model by Rao and Xiao (2012), show similar types of clustering merely due to Mexican-hat connectivity. One possibility would be that in many hierarchical unsupervised neural network models, each layer is trained separately in turn. This is important for synaptic connectivities in higher layers to be appropriately tuned. However, in our model, all the synaptic connectivities are learned simultaneously, which may be more realistic. The implication would be that STDP may allow a network to learn connectivities more flexibly without the traditional greedy method of teaching one layer at a time. We propose this hypothesis because adding another dimension of timing via STDP allows the synaptic weights to be dynamically updated in realtime whereas rate coded neurons depend on averaged firing rates within pre-specified time windows.
Furthermore, investigating neuronal voltage dynamics revealed the presence of both ON-cells and OFF-cells, which respond maximally immediately after presentation or removal of a particular color input. These results led us to hypothesize that the emergence of OFF-cells was caused by different spike timing delays from input cells (section 3.3).

The role of neuronal synchrony in color processing is still an open question particularly since our model demonstrates that information analysis based on firing rates can successfully predict the color input. However, while the network was trained with various color input in natural images, in this analysis, the network was tested only with eight clearly distinct colors, and in order to accurately decode the subtle differences between similar colors, synchrony and its timing may play an important role for the representations at least in our proposing mechanism. In addition, the importance of timing delays in the creation of ON/OFF cells suggests rate codes alone may not be sufficient in visual system development.

\section{ROLE OF SPIKE-TIMING DELAYS IN CREATING ON/OFF CELLS}

The mechanism of the emergence of OFF-cells due to spike timing delays allows us to propose a possible in vivo mechanism of the development of the ON/OFF-cell that is also combined with the R/G opponency shown in Figure 9. As shown in Figure 11, we suppose there is a simplified network that consists of three cells in the LGN layers and two cells in output layer $\left(R_{\mathrm{ON}} / G_{\mathrm{OFF}}\right.$ cell and its neighboring cell $\mathrm{N}$ ). In this schematic, LGN cells consist of a $\mathrm{C} 1$ (R/G opponent) cell and two L (monochrome) cells. In addition, one of the $\mathrm{L}$ cells, $\mathrm{L}_{1}$, has a delayed Green input (see details in Figure 10).

When the color red is presented to the network (Figure 11A), all three cells in the LGN become activated, and the $R_{\mathrm{ON}} / G_{\mathrm{OFF}}$ output cell that receives excitatory inputs from the $\mathrm{C} 1$ cell and one $\mathrm{L}$ cell $\left(\mathrm{L}_{1}\right)$ becomes highly activated. When the red input is removed (Figure 11B), only L cells become slightly activated due to the delayed connection, which does not have a large influence on the $R_{\mathrm{ON}} / G_{\mathrm{OFF}}$ cell.

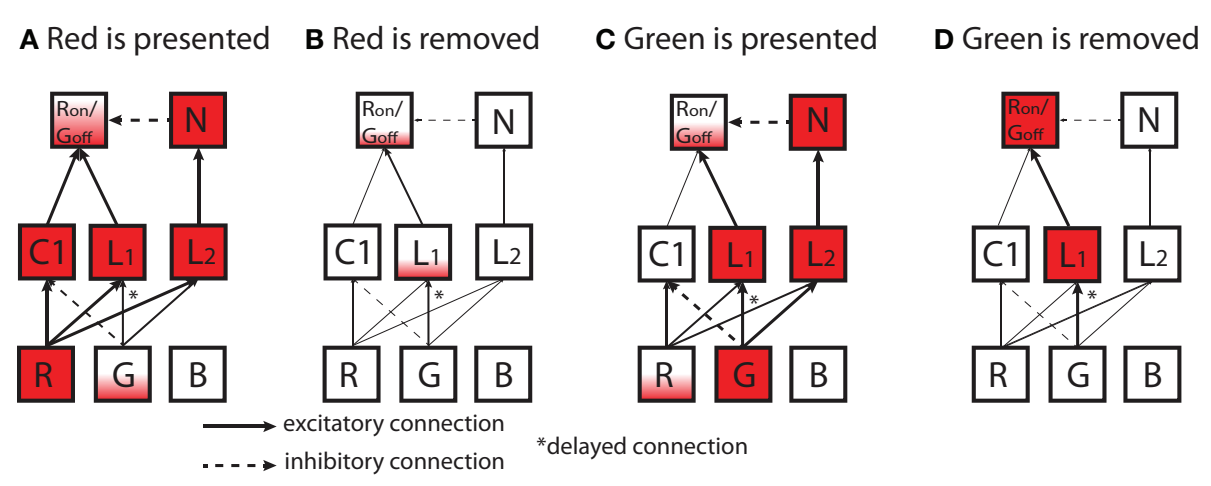

FIGURE 11 | Diagrams of a simplified network to explain emergence of ON/OFF-cells that is also combined with $R / G$ opponency shown in Figure 9. Each box represents a neuron. Inputs leading to the activation/inactivation of a $R_{\mathrm{ON}} / G_{\mathrm{OFF}}$ output cell is shown ((A) red is presented; (B) red is removed; (C) green is presented; (D) green is removed). Here, $\mathrm{N}$ represents the neighboring cell of the $R_{\mathrm{ON}} / G_{\mathrm{OFF}}$ output cell. In the intermediate layer, $\mathrm{C} 1$ is a cell in the Red/Green color opponent channel (R $G$ ) and $L$ is a cell in the luminance channel $(R+G)$. In the input layer on the bottom, each R, G, B represent a photoreceptor. Neurons are filled in proportion to their activation level. Solid (broken) arrows represent excitatory (inhibitory) connections. A star placed next to an arrow means that the connection has delayed synaptic timing as discussed in Figure 10. 
When Green color input is presented to the same network (Figure 11C), the L cells become activated. Subsequently, the N cell in the output layer that sends an inhibitory signal to the $R_{\mathrm{ON}} / G_{\mathrm{OFF}}$ cell becomes activated as well. Because of the inhibition, the $R_{\mathrm{ON}} / G_{\mathrm{OFF}}$ cell does not become highly activated even though it receives excitatory input from the preceding $\mathrm{L}_{1}$ cell. When the color input is removed (Figure 11D), the $\mathrm{L}_{1}$ cell that has the delayed connection from the Green cell is kept activated, which causes the $R_{\mathrm{ON}} / G_{\mathrm{OFF}}$ cell to become activated.

Similarly, a possible mechanism of the ON/OFF-cell that is combined with Y/B opponency is provided in Figure 12. In the figure, we suppose a simplified network consists of three C2 (Y/B opponent) cells in the LGN layer and three cells in the output layer $\left(Y_{\mathrm{ON}} / B_{\mathrm{OFF}}, \mathrm{N}_{1}\right.$, and $\left.\mathrm{N}_{2}\right)$. Each cell in the output layer receives excitatory input from one $\mathrm{C} 2$ cell $\left(\mathrm{C} 22_{1}, \mathrm{C} 22_{2}\right.$, and $\left.\mathrm{C} 22_{3}\right)$ cell). $\mathrm{N}_{2}$ cell establishes inhibitory connection to $\mathrm{N}_{1}$, and the $\mathrm{N}_{1}$ establishes an excitatory connection to the target cell, $Y_{\mathrm{ON}} / B_{\mathrm{OFF}}$. In this network, $\mathrm{C}_{2}$ cell establishes the delayed connections discussed above (in Figure 10) from the R and $\mathrm{G}$ cells, and $\mathrm{C}_{3}$ cell establishes delayed connections from all R, G, and B cells.

As shown in Figure 12A, when the color yellow is presented, all $\mathrm{C} 2$ cells become activated. As a result, the target cell, $Y_{\mathrm{ON}} / B_{\mathrm{OFF}}$, should become highly activated by receiving excitatory input from the preceding $\mathrm{C} 22_{1}$ cell. In addition, the target cell $Y_{\mathrm{ON}} / B_{\mathrm{OFF}}$ receives some excitation from $\mathrm{N}_{1}$ cell. When the color input is removed (Figure 12B), due to the delayed connections, $\mathrm{C} 22_{2}$ and $\mathrm{C} 22_{3}$ cells are kept active for an interval, but not $\mathrm{C} 2{ }_{1}$. As a result, the target cell, $Y_{\mathrm{ON}} / B_{\mathrm{OFF}}$ would not get highly activated.

When the color blue is presented (Figure 12C), none of the C2 cells would become activated, leading to no activation of the target cell. On the other hand, when the color input is removed (Figure 12D), C2 2 cell becomes activated to some degree due to the delayed connection from $\mathrm{R}$ and $\mathrm{G}$ with their weak activations caused by the color blue. This leads to the activation of $\mathrm{N}_{1}$ that establishes excitatory connection to the target cell, $Y_{\mathrm{ON}} / B_{\mathrm{OFF}}$. In this way, it is possible to provide a possible dynamical mechanism of ON/OFF-cells that involves color opponency.

In order to test the hypothesized architectures above, we have modeled the simple networks using the same set of neurons used in our computational model and recorded firing activity of each neuron for $300 \mathrm{~ms}$ ( $240 \mathrm{~ms}$ of color input presentation followed by $60 \mathrm{~ms}$ of no color input presentation) (Figure 13). The results show that the same target neuron exhibits characteristics of both $R_{\mathrm{ON}} / G_{\mathrm{OFF}}$, and $Y_{\mathrm{ON}} / B_{\mathrm{OFF}}$ firing activity. However, the result also showed that those responses are not observed immediately after the presentation or removal of the color input. In other words, there is still activity in ON-cells after the stimulus is turned off. Also, OFF-cells show responses when the stimulus is turned on. These effects are due to the transitional delay of signals. However, as shown in Figures 8, 9, the population activity shows a more clear ON/OFF response.

\section{POTENTIAL LIMITATIONS}

Although our model predicts that spike timing is important for the effective development of color selectivity, our model did not investigate development of orientation selectivity, which is known to coexist with color selectivity, as investigated in previous models (Barrow et al., 1996; Rao and Xiao, 2012). Therefore, in future work it will be important to model co-development of both color and orientation selectivity. A different limitation of our model is that the representation of color input was based on simplified input cells that detect digital RGB values. To investigate more realistic mechanisms of development, biologically-accurate architectures of the various types of retinal cells that are involved in the process should be implemented.

\section{CONVERGENCE OF APPROACHES}

Our model of the early visual system displays convergence between the fields of computational neuroscience and artificial neural networks (ANNs). Computational neuroscience has

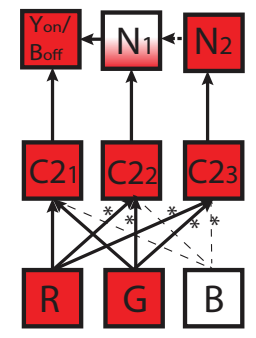

$\longrightarrow$ ... inhibitory connection

FIGURE 12 | Diagrams of simplified network to explain a possible mechanism of ON/OFF-cell that is combined with the Y/B opponency reported in Figure 9. In the figure, each box represents a cell in the network. Inputs leading to the activation/inactivation of a $Y_{\mathrm{ON}} / B_{\mathrm{OFF}}$ output cell are shown: ((A) yellow is presented; (B) yellow is removed; (C) blue is presented; (D) blue is removed). In the output layer on the top, the target cell shown as $Y_{\mathrm{ON}} / B_{\mathrm{OFF}}$ in each box represents the ON/OFF target cell, $N_{1}$ is a neighboring cell that sends excitatory connections to $Y_{\mathrm{ON}} / B_{\mathrm{OFF}}$, and $\mathrm{N}_{2}$ is a cell that

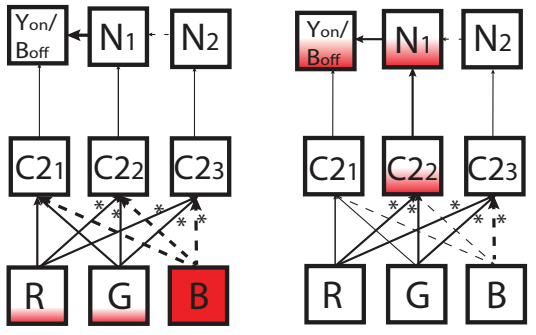

*delayed connection

sends excitatory connections to $N_{1}$. In the intermediate layer, $\mathrm{C} 2$ is a cell in the Yellow/Blue color opponent channel $[(R+G)-B]$. In the input layer on the bottom, each R, G, B represent each photoreceptor. Cells are filled with a color to provide degrees of activations of different cells; partially filled box means it is only activated a small amount. All solid arrows represent excitatory connections while broken arrows represent inhibitory connections. A star placed next to an arrow means that the connection has a delayed synaptic timing as discussed in Figure 10. 


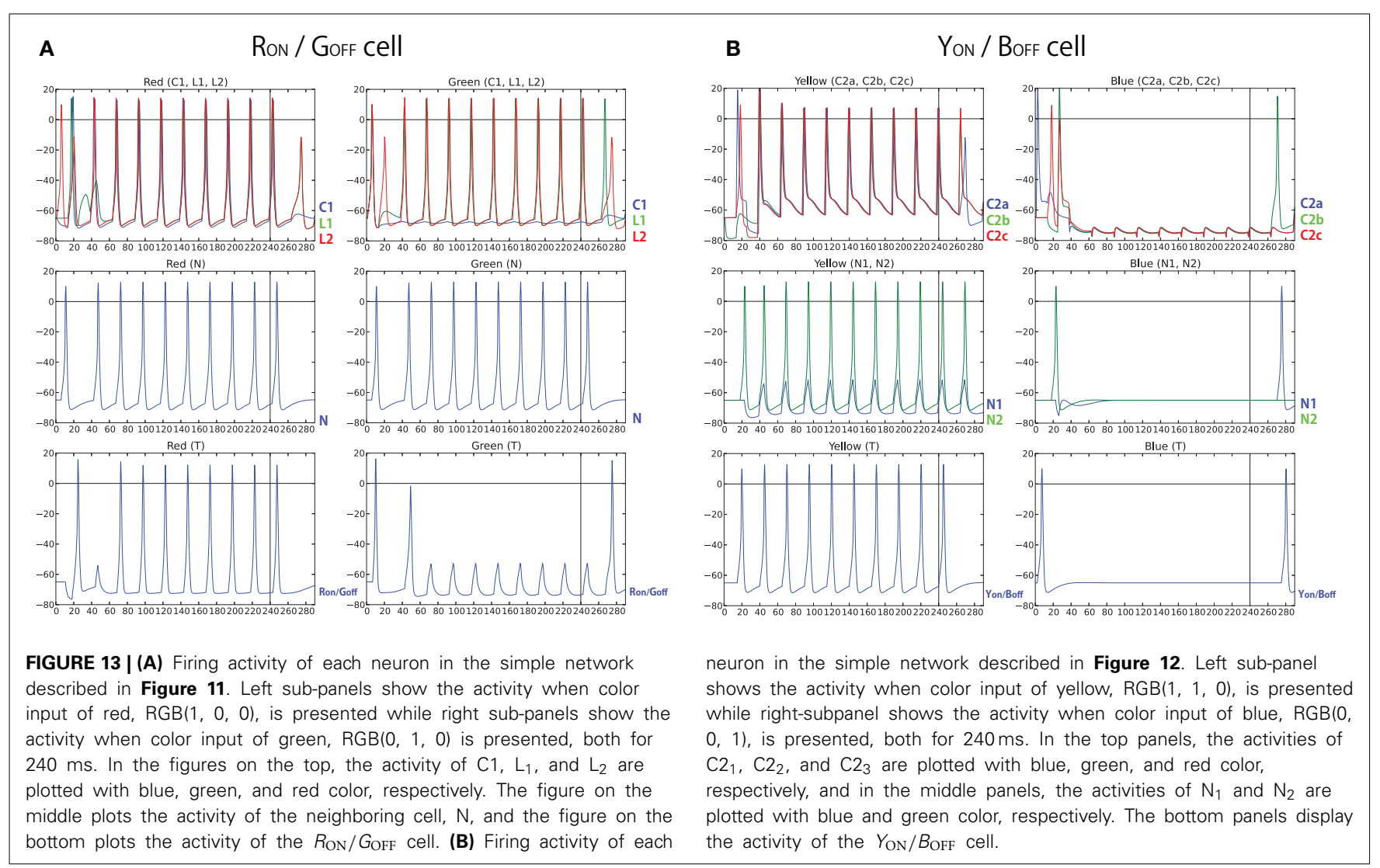

traditionally attempted to understand neuronal dynamics by building models by using known biological detail without forcing an explicit engineered goal. ANNs, which emerged from the field of artificial intelligence, have stressed an approach that aims to develop systems displaying intelligence by constraining the system design to a specified goal, while taking inspiration from biological systems (Hinton et al., 2006).

Recent developments in ANNs, including deep learning, a technique drawing inspiration from neurobiology, have made significant progress in recent years (Hinton et al., 2006) improving performance on visual information processing (Lee et al., 2009). Progress has also been made by training recurrent neural networks to perform extremely well on difficult, specialized classes of problems, such as handwritten character recognition (Graves and Schmidhuber, 2008). Related developments have also started focusing on investigations into utilizing brain-inspired informatics to improve the intelligence of current technologies (Eguchi et al., 2013). However, currently, even the best machine learning algorithms have difficulty in matching human performance in recognizing arbitrary classes of complex visual stimuli. Basic research in neurobiology, combined with utilization of biological detail in computer models, is therefore needed to enable further improvements in machine learning. Improved understanding of how the brain circuitry represents and processes visual information may inspire new classes of visual processing algorithms. We have used this approach to design our model, which allows correlation of its neuronal dynamics with electrophysiological data, takes into account known neuroanatomy, and uses a biologically

plausible learning rule (Markram et al., 1997), and therefore takes a step toward improved understanding of in vivo brain dynamics.

\section{NEOCORTICAL ARCHITECTURE}

One of the basic goals of neuroscience is to elucidate the mechanisms by which the structure of the brain leads to its function (Shepherd, 2004). This depends on a careful study of neuroanatomy as well as functional measures in vivo (Weiler et al., 2008). The importance of changes in microcircuitry is underscored with experimental studies that have shown how alterations in cortical connectivity can lead to diseases, such as autism (Qiu et al., 2011). Since it is not possible to measure the state of all neurons it is important to combine computer modeling with known neurophysiological circuitry data (Lytton, 2008). Following this approach in our model allows us to make predictions on the function and development of several features observed in visual cortex in vivo.

Our model suggests that in vivo, the process of development of color clustering is more likely to initiate in earlier layers (L4) of V1. This may be testable via electrophysiological methods applied during different stages of development. Our model is also consistent with more general implications, suggesting that through a process of development, each layer of neocortex may learn to enhance important signals as they progress within the microcircuitry. Although initial synaptic weights in our model were randomly distributed, visual information and STDP allowed the feed-forward projections of the neocortex to learn the color information as the signals flowed in successive layers. In our model, the 
color information progressed from $\mathrm{L} 4 \rightarrow \mathrm{L} 2 / 3 \rightarrow \mathrm{L} 5$. Although L4 is the input layer into V1, the final output layer (L5) had the highest information content about the color stimuli. Further experiments will be needed to elucidate the role that individual layers play in shaping the information coding capacity of the neocortex.

Prior modeling (Stringer and Rolls, 2002; Rolls and Stringer, 2006; Dura-Bernal et al., 2012) and experiments (Hung et al., 2005) have shown the importance of the feed-forward architecture of the visual cortex ventral stream for object recognition. Although our work makes use of the feed-forward architecture of cortical areas, it also takes into account additional details of wiring, including recurrent connectivity. As more microcircuitry data becomes available, it will be possible to refine our model further (Alivisatos et al., 2013). Part of this process will involve combined experimental/computational approaches. For example, Hung et al. (2005) studied the ventral visual pathway with the aim of understanding how object recognition takes place by building pattern recognition algorithms that utilize inferotemporal cortex neuronal spiking information to assess both object category and identity. In the future it will be possible to extend our model to use similar techniques to quantify performance in object recognition that is based on accurate color processing.

\section{ACKNOWLEDGMENTS}

The authors would like to thank Erik De Schutter (Okinawa Institute of Science and Technology) for organizing the Okinawa Computational Neuroscience Course 2013, where some of this research was conducted; Michael Hines (Yale) and Ted Carnevale (Yale) for NEURON simulator support; Tom Morse (Yale) for ModelDB support.

\section{REFERENCES}

Abbott, L. F., and Nelson, S. B. (2000). Synaptic plasticity: taming the beast. Nat. Neurosci. 3, 1178-1183. doi: 10.1038/78829

Adesnik, H., and Scanziani, M. (2010). Lateral competition for cortical space by layer-specific horizontal circuits. Nature 464, 1155-1160. doi: 10.1038 /nature08935

Alivisatos, A., Andrews, A., Boyden, E., Chun, M., Church, G., Deisseroth, K., et al. (2013). Nanotools for neuroscience and brain activity mapping. Acs Nano. 7, 1850-1866. doi: 10.1021/nn4012847

Barbour, B., Brunel, N., Hakim, V., and Nadal, J.-P. (2007). What can we learn from synaptic weight distributions? Trends Neurosci. 30, 622-629. doi: 10.1016/j.tins.2007.09.005

Barrow, H. G., Bray, A. J., and Budd, J. M. (1996). A self-organizing model of "color blob" formation. Neural Comput. 8, 1427-1448. doi: 10.1162/neco.1996.8.7.1427

Basalyga, G., Gleiser, P. M., and Wennekers, T. (2011). "Emergence of small-world structure in networks of spiking neurons through STDP plasticity," in From Brains to Systems. Advances in experimental medicine and biology, Vol. 718, eds C. Hernández, R. Sanz, J. Gómez-Ramirez, L. S. Smith, A. Hussain, A. Chella, et al. (New York, NY: Springer), 33-39.

Bednar, J. A., De Paula, J. B., and Miikkulainen, R. (2005). Self-organization of color opponent receptive fields and laterally connected orientation maps. Neurocomputing 65-66, 69-76. doi: 10.1016/j.neucom.2004.10.055

Bi, G. Q., and Poo, M. M. (1998). Synaptic modifications in cultured hippocampal neurons: dependence on spike timing, synaptic strength, and postsynaptic cell type. J. Neurosci. 18, 10464-10472.

Carnevale, N. T., and Hines, M. L. (2009). The Neuron Book. Cambridge: Cambridge University Press.

Casagrande, V. (1994). A third parallel visual pathway to primate area v1. Trends Neurosci. 17, 305-310. doi: 10.1016/0166-2236(94)90065-5
Chatterjee, S., and Callaway, E. M. (2003). Parallel colour-opponent pathways to primary visual cortex. Nature 426, 668-671. doi: 10.1038/nature02167

Choe, Y., and Miikkulainen, R. (1998). Self-organization and segmentation in a laterally connected orientation map of spiking neurons. Neurocomputing 21, 139-158. doi: 10.1016/S0925-2312(98)00040-X

Douglas, R. J., and Martin, K. A. C. (2007). Mapping the matrix: the ways of neocortex. Neuron 56, 226-238. doi: 10.1016/j.neuron.2007.10.017

Dura-Bernal, S., Wennekers, T., and Denham, S. L. (2012). Top-down feedback in an hmax-like cortical model of object perception based on hierarchical bayesian networks and belief propagation. PLOS ONE 7:e48216. doi: 10.1371/journal.pone.0048216

Eguchi, A., Nguyen, H., and Thompson, C. (2013). Everything is alive: towards the future wisdom Web of things. World Wide Web 16, 357-378. doi: 10.1007/s11280-012-0182-4

Evans, B. D., and Stringer, S. M. (2013). How lateral connections and spiking dynamics may separate multiple objects moving together. PLOS ONE 8:e69952. doi: 10.1371/journal.pone.0069952

Freeman, J., and Simoncelli, E. P. (2011). Metamers of the ventral stream. Nat. Neurosci. 14, 1195-1201. doi: 10.1038/nn.2889

Freiwald, W. A., and Tsao, D. Y. (2010). Functional compartmentalization and viewpoint generalization within the macaque face-processing system. Science 330, 845-851. doi: 10.1126/science.1194908

Friedman, H. S., Zhou, H., and Heydt, R. V. D. (2003). The coding of uniform colour figures in monkey visual cortex. J. Physiol. 548, 593-613. doi: 10.1113/jphysiol.2002.033555

Gerstner, W., Kempter, R., van Hemmen, J. L., and Wagner, H. (1996). A neuronal learning rule for sub-millisecond temporal coding. Nature 383, 76-78. doi: 10.1038/383076a0

Goda, N., Koida, K., and Komatsu, H. (2009). "Colour representation in lateral geniculate nucleus and natural colour distributions," in Computational Color Imaging, Lecture notes in computer science, Vol. 5646, eds Trémeau, A., Schettini, R., and Tominaga, S. (Berlin: Springer), 23-30.

Graves, A., and Schmidhuber, J. (2008). "Offline handwriting recognition with multidimensional recurrent neural networks," in Advances in Neural Information Processing Systems 21, eds D. Koller, D. Schuurmans, Y. Bengio and L. Bottou (Morehouse Lane Red Hook, NY: Curran Associates, Inc.), 545-552.

Hines, M., Morse, T., Migliore, M., Carnevale, N., and Shepherd, G. (2004). ModelDB: a database to support computational neuroscience. J. Comput. Neurosci. 17, 7-11. doi: 10.1023/B:JCNS.0000023869.22017.2e

Hines, M. L., and Carnevale, N. T. (2001). NEURON: a tool for neuroscientists. Neuroscientist 7, 123-135. doi: 10.1177/107385840100700207

Hines, M. L., Davison, A. P., and Muller, E. (2009). NEURON and python. Front. Neuroinform. 3:1. doi: 10.3389/neuro.11.001.2009

Hinton, G., Osindero, S., and Teh, Y. (2006). A fast learning algorithm for deep belief nets. Neural Comput. 18, 1527-1554. doi: 10.1162/neco.2006.18.7.1527

Hopf, J., Boehler, C., Luck, S., Tsotsos, J., Heinze, H., and Schoenfeld, M. (2006). Direct neurophysiological evidence for spatial suppression surrounding the focus of attention in vision. Proc. Natl. Acad. Sci. U.S.A. 103, 1053-1058. doi: 10.1073/pnas.0507746103

Hubel, D. H., and Wiesel, T. N. (1962). Receptive fields, binocular interaction and functional architecture in the cat's visual cortex. J. Physiol. 160, 106-154.2.

Hung, C., Kreiman, G., Poggio, T., and DiCarlo, J. (2005). Fast readout of object identity from macaque inferior temporal cortex. Science 310, 863. doi: $10.1126 /$ science. 1117593

Kang, K., Shelley, M., and Sompolinsky, H. (2003). Mexican hats and pinwheels in visual cortex. Proc. Natl. Acad. Sci. U.S.A. 100, 2848-2853. doi: $10.1073 /$ pnas.0138051100

Kato, H., Ikeguchi, T., and Aihara, K. (2009). "Structural analysis on STDP neural networks using complex network theory," in Artificial Neural Networks ICANN 2009. Lecture notes in computer science, Vol. 5768, eds C. Alippi, M. Polycarpou, C. Panayiotou, and G. Ellinas (Springer Berlin), 306-314. doi: 10.1007/978-3-642-04274-4_32

Kato, H., Kimura, T., and Ikeguchi, T. (2007). "Emergence of self-organized structures in a neural network using two types of STDP learning rules," in Proceedings of 2007 International Symposium on Nonlinear Theory and its Applications (Vancouver, Canada), 429-432.

Komatsu, H. (1998). Mechanisms of central color vision. Curr. Opin. Neurobiol. 8, 503-508. doi: 10.1016/S0959-4388(98)80038-X 
Komatsu, H., and Goda, N. (2009). Color information processing in higher brain areas. in Computational Color Imaging. Lecture notes in computer science, Vol. 5646, eds A. Trémeau, R. Schettini, and S. Tominaga (Berlin: Springer), 1-11. doi: 10.1007/978-3-642-03265-3_1

Landisman, C. E., and Ts'o, D. Y. (2002). Color processing in macaque striate cortex: electrophysiological properties. J. Neurophysiol. 87, 3138-3151. doi: 10.1152/jn.00957.1999

Lee, H., Grosse, R., Ranganath, R., and Ng, A. (2009). “Convolutional deep belief networks for scalable unsupervised learning of hierarchical representations," in Proceedings of the 26th Annual International Conference on Machine Learning (New York, NY: ACM), 609-616.

Livingstone, M. S., and Hubel, D. H. (1984). Anatomy and physiology of a color system in the primate visual cortex. J. Neurosci. 4, 309-356.

Lu, H. D., and Roe, A. W. (2008). Functional organization of color domains in v1 and v2 of macaque monkey revealed by optical imaging. Cereb. Cortex 18, 516-533. doi: 10.1093/cercor/bhm081

Lytton, W. (2008). Computer modelling of epilepsy. Nat. Rev. Neurosci. 9, 626-637. doi: 10.1038/nrn2416

Markram, H., Lübke, J., Frotscher, M., and Sakmann, B. (1997). Regulation of synaptic efficacy by coincidence of postsynaptic APs and EPSPs. Science 275, 213-215. doi: 10.1126/science.275.5297.213

Martin, K. (2002). Microcircuits in visual cortex. Curr. Opin. Neurobiol. 12, 418-425. doi: 10.1016/S0959-4388(02)00343-4

Michael, C. R. (1978). Color vision mechanisms in monkey striate cortex: dual-opponent cells with concentric receptive fields. J. Neurophysiol. 41, 572-588.

Neymotin, S., Chadderdon, G., Kerr, C., Francis, J., and Lytton, W. (2013). Reinforcement learning of 2-joint virtual arm reaching in computer model of sensorimotor cortex. Neural Comput. 25, 3263-3293. doi: 10.1162/NECO_a_00521

Neymotin, S., Kerr, C., Francis, J., and Lytton, W. (2011a). “Training oscillatory dynamics with spike-timing-dependent plasticity in a computer model of neocortex," in 2011 IEEE Signal Processing in Medicine and Biology Symposium (SPMB) (New York, NY), 1-6. doi: 10.1109/SPMB.2011.6120115

Neymotin, S., Lee, H., Park, E., Fenton, A., and Lytton, W. (2011b). Emergence of physiological oscillation frequencies in a computer model of neocortex. Front. Comput. Neurosci. 5:19. doi: 10.3389/fncom.2011.00019

Paik, S.-B., and Ringach, D. L. (2011). Retinal origin of orientation maps in visual cortex. Nat. Neurosci. 14, 919-925. doi: 10.1038/nn.2824

Pettet, M. W., and Gilbert, C. D. (1992). Dynamic changes in receptive-field size in cat primary visual cortex. Proc. Natl. Acad. Sci. U.S.A. 89, 8366-8370. doi: 10.1073/pnas.89.17.8366

Qiu, S., Anderson, C., Levitt, P., and Shepherd, G. (2011). Circuit-specific intracortical hyperconnectivity in mice with deletion of the autismassociated Met receptor tyrosine kinase. J. Neurosci. 31, 5855-5864. doi: 10.1523/JNEUROSCI.6569-10.2011

Quattoni, A., and Torralba, A. (2009). "Recognizing indoor scenes," in IEEE Conference on Computer Vision and Pattern Recognition, 2009. CVPR 2009 (Miami, FL: IEEE), 413-420. doi: 10.1109/CVPR.2009.5206537

Rao, A., and Xiao, Y. (2012). "A computational model of early visual cortex using konio-cellular pathway projections," in The 2012 International Joint Conference on Neural Networks (IJCNN) (Brisbane, QLD), 1-8.

Rolls, E. T., and Milward, T. (2000). A model of invariant object recognition in the visual system: learning rules, activation functions, lateral inhibition, and information-based performance measures. Neural Comput. 12, 2547-2572. doi: 10.1162/089976600300014845

Rolls, E. T., and Stringer, S. M. (2006). Invariant visual object recognition: a model, with lighting invariance. J. Physiol. Paris 100, 43-62. doi: 10.1016/j.jphysparis.2006.09.004

Rolls, E. T., and Treves, A. (1998). Neural Networks and Brain Function. Oxford, NY: Oxford University Press.
Rowan, M., and Neymotin, S. (2013). "Synaptic scaling balances learning in a spiking model of neocortex," in Adaptive and Natural Computing Algorithms, eds M. Tomassini, A. Antonioni, F. Daolio, and P. Buesser (Berlin: Springer), 20-29.

Rowe, M. H. (2002). Trichromatic color vision in primates. News Physiol. Sci. 17, 93-98. doi: 10.1152/nips.01376.2001

Royer, S., and Paré, D. (2003). Conservation of total synaptic weight through balanced synaptic depression and potentiation. Nature 422, 518-522. doi: 10.1038 /nature 01530

Rullen, R. V., and Thorpe, S. J. (2001). Rate coding versus temporal order coding: what the retinal ganglion cells tell the visual cortex. Neural Comput. 13, 1255-1283. doi: 10.1162/08997660152002852

Salzmann, M. F. V., Bartels, A., Logothetis, N. K., and Schü, A. (2012). Color blobs in cortical areas v1 and $\mathrm{v} 2$ of the new world monkey Callithrix jacchus, revealed by non-differential optical imaging. J. Neurosci. 32, 7881-7894. doi: 10.1523/JNEUROSCI.4832-11.2012

Shapley, R., Kaplan, E., and Soodak, R. (1981). Spatial summation and contrast sensitivity of $\mathrm{x}$ and $\mathrm{y}$ cells in the lateral geniculate nucleus of the macaque. Nature 292, 543-545. doi: 10.1038/292543a0

Shepherd, G. (2004). The Synaptic Organization of the Brain. Oxford, NY: Oxford University Press. doi: 10.1093/acprof:oso/9780195159561.001.1

Shin, C.-W., and Kim, S. (2006). Self-organized criticality and scale-free properties in emergent functional neural networks. Phys. Rev. E 74, 045101. doi: 10.1103/PhysRevE.74.045101

Somers, D. C., Nelson, S. B., and Sur, M. (1995). An emergent model of orientation selectivity in cat visual cortical simple cells. J. Neurosci. 15, 5448-5465.

Song, S., Miller, K. D., and Abbott, L. F. (2000). Competitive hebbian learning through spike-timing-dependent synaptic plasticity. Nat. Neurosci. 3, 919-926. doi: $10.1038 / 78829$

Stringer, S. M., and Rolls, E. T. (2002). Invariant object recognition in the visual system with novel views of 3D objects. Neural Comput. 14, 2585-2596. doi: $10.1162 / 089976602760407982$

Sugase, Y., Yamane, S., Ueno, S., and Kawano, K. (1999). Global and fine information coded by single neurons in the temporal visual cortex. Nature 400, 869-873. doi: $10.1038 / 23703$

Weiler, N., Wood, L., Yu, J., Solla, S., and Shepherd, G. (2008). Top-down laminar organization of the excitatory network in motor cortex. Nat. Neurosci. 11, 360-366. doi: 10.1038/nn2049

Xiao, Y., Wang, Y., and Felleman, D. J. (2003). A spatially organized representation of colour in macaque cortical area v2. Nature 421, 535-539. doi: 10.1038/nature01372

Yu, S., Huang, D., Singer, W., and Nikoli, D. (2008). A small world of neuronal synchrony. Cereb. Cortex 18, 2891-2901. doi: 10.1093/cercor/bhn047

Conflict of Interest Statement: The authors declare that the research was conducted in the absence of any commercial or financial relationships that could be construed as a potential conflict of interest.

Received: 22 October 2013; accepted: 17 February 2014; published online: 12 March 2014.

Citation: Eguchi A, Neymotin SA and Stringer SM (2014) Color opponent receptive fields self-organize in a biophysical model of visual cortex via spike-timing dependent plasticity. Front. Neural Circuits 8:16. doi: 10.3389/fncir.2014.00016

This article was submitted to the journal Frontiers in Neural Circuits.

Copyright (c) 2014 Eguchi, Neymotin and Stringer. This is an open-access article distributed under the terms of the Creative Commons Attribution License (CC BY). The use, distribution or reproduction in other forums is permitted, provided the original author(s) or licensor are credited and that the original publication in this journal is cited, in accordance with accepted academic practice. No use, distribution or reproduction is permitted which does not comply with these terms. 